\section{La nutrición: un aspecto importante en la calidad de vida de la mujer}

\section{Nutrition: an important aspect in women's quality of life}

Goliat Reina Gómez 1

Berta R. Rodríguez Anzardo 2

1 Hospital Ginecobstétrico Ramón González Coro. Calle 21 n. 856 entre 4 y 6 Vedado. Municipio Plaza de la Revolución. Ciudad de La Habana, Cuba. Código Postal 10400.

2 Instituto Nacional de Endocrinología, Ciudad de La Habana, Cuba

\begin{abstract}
The preamble of the 53 rd session of the Commission for Human Rights included the right to an adequate quality of life including nutrition. There has been a long-standing concern of international organizations for the existence of such quality and the possibilities to acquire it. Some bad perinatal results are due to poor and unbalanced diets that may be corrected by nutritional education and social promotion through the analysis of food habits, available food and access to food. The efforts to solve this problem are related to food security and social pressure towards development with justice at the world level.

Key words Nutrition disorders, Feed, Nutritive value, Food quality, Cultural factors
\end{abstract}

Resumo La $53^{a}$ sesión de la Comisión de Derechos Humanos, incluía en su preámbulo el derecho a un nivel de vida adecuado, incluyendo la alimentación. Desde hace años existe una gran preocupación por los organismos internacionales en relación con la producción de alimentos, por la gravedad y extensión del problema y su impacto económico. La accesibilidad de los alimentos está determinada por la existencia de los mismos y las posibilidades de adquisición. Algunos malos resultados perinatales son producto de una dieta insuficiente y desequilibrada, que puede corregirse utilizando la educación nutricional y la promoción social, analizando los hábitos alimentarios, los alimentos disponibles y su accesibilidad. Los esfuerzos para la solución de este problema están relacionados con la seguridad alimentaria y la presión social que se ejerza para lograr la posibilidad de un desarrollo armónico a escala mundial.

Palavras-chave Trastornos nutricionales, Alimentación, Valor nutritivo, Calidad de los alimentos, Factores culturais 


\section{Introducción}

El crecimiento normal de las especies animales superiores está determinado por factores genéticos y nutricionales, principalmente. La nutrición es un elemento tempranamente reconocido por el hombre como factor de riesgo para la salud, pues la colección hipocrática ya incluye preocupaciones acerca de la alimentación y el modo de utilizar los productos alimentarios para evitar enfermedades.

Las carencias alimentarias cuantitativas o cualitativas en la mujer tienen una especial significación, tanto por el aumento de la morbimortalidad de ésta, como por las consecuencias directas sobre el crecimiento y desarrollo fetal, afectando de manera previsible el futuro de la especie humana. Es bien conocido que el peso medio de los recién nacidos en países menos desarrollados es significativamente más bajo que en los países subdesarrollados, con mayor riesgo para la supervivencia. 1

\section{Desarrollo}

La 53 ${ }^{\mathrm{a}}$ sesión de la Comisión de Derechos Humanos, celebrada entre el 10 de marzo y el 18 de abril de 1997 en Ginebra, aprobó una resolución sobre el derecho a la alimentación, ${ }^{2}$ en cuyo preámbulo se incluía el derecho a un nivel de vida adecuado, incluyendo la alimentación, lo cual se expresa en la Declaración Universal de los Derechos Humanos, así como referencias a la Declaración Universal sobre la Erradicación del Hambre y la Malnutrición y al Convenio Internacional sobre Derechos Económicos, Sociales y Culturales.

Tradicionalmente se ha relacionado a la malnutrición e infección con pobreza y a la obesidad y las enfermedades no transmisibles con el bienestar económico, esquema que se ha visto modificado en los últimos años, al apreciarse una tendencia a la convergencia de todos los elementos en los más desposeí$\operatorname{dos}^{3} \mathrm{y}$ en particular en la mujer, como le demuestra un estudio del año 2000 realizado en Cuba, donde se comprueba $20,5 \%$ de obesidad y $7,5 \%$ de desnutrición en grupos del sexo masculino contra $25,4 \%$ y $13,6 \%$ de incidencia en el sexo femenino. 4

Se reconocen desde hace años como problemas de salud importantes en el mundo actual las afecciones cardiovasculares, la hipertensión y la diabetes Mellitus, donde aparece la nutrición como elemento de causalidad: dietas bajas en proteínas y fibra dietética, 5,6 contenido de sal, ${ }^{7}$ niveles plasmáticos de ácido ascórbico, 8 papel del calcio en la regulación insulínica ${ }^{9}$ y la ingestión de otros cationes $(\mathrm{K}, \mathrm{Mg}) .{ }^{10}$
Lo relativamente nuevo es que la tradicional prevalencia masculina de estas enfermedades ha dejado de existir, al menos en Cuba. ${ }^{11}$

Indiscutiblemente la malnutrición materna condiciona problemas carenciales que afectan la posibilidad de supervivencia del feto y su futuro desarrollo psicomotor, algo directamente relacionado con las posibilidades de conservación de la especie humana.12-14 Las patologías asociadas con el embarazo y la nutrición también producen un aumento de los gastos de hospitalización, como es el caso de los trastornos hipertensivos en el embarazo, los cuales se asocian en muchos casos a deficiencias nutricionales especificas que incluyen la riboflavina $15 \mathrm{o}$ la susceptibilidad para sufrir modificaciones oxidativas vinculadas a los niveles séricos de lipoproteínas. 16

No puede ignorarse el impacto económico de la malnutrición femenina porque ésta constituye gran parte de la fuerza laboral en el mundo, como ocurre en Africa, repercutiendo sobre la capacidad de producción, incluida la alimentaria. En los países latinoamericanos, donde la mujer es frecuentemente la cabeza de familia, la afectación materna constituye la principal causa de disrrupción familiar, reconociendo que la mujer, en su condición de "administradora familiar" y proveedora primaria de cuidados, es la más afectada, sacrificando sus posibilidades de alimentarse adecuadamente a favor de los niños y ancianos bajo su guarda.

Los esfuerzos para la solución de este problema están relacionados con las posibilidades de lograr la seguridad alimentaria y con la presión social que se ejerza para lograr la posibilidad de un desarrollo armónico a escala mundial.

\section{Disponibilidad de alimentos}

Desde hace años existe una gran preocupación por los organismos internacionales en relación con la producción de alimentos. En 1985 la Food and Agriculture Organization (FAO), un organismo de las Naciones Unidas, estableció las necesidades de energías y proteínas para prevenir la desnutrición proteico calórica en la población general.17 Las recomendaciones cubanas de 1996 se establecieron con un margen de seguridad mayor que el propuesto por el Comité de Expertos. ${ }^{18}$

¿Qué factores condicionan la disponibilidad de los alimentos diez años después de la Cumbre de Río? Entre varios, podemos citar el deterioro del medio ambiente, con el incremento de la sequía y la desertificación, 19 que afectan severamente al continente africano y los cambios climáticos globales, producto 
del efecto invernadero y la emisión de $\mathrm{CO}$, pese a la existencia del Convenio de Kioto, aún no aceptado por los EEUU, el principal país contaminante. 20

La disminución de áreas de siembra por razones comerciales (política que ha seguido la Unión Europea en algunos cultivos) y la disponibilidad de semillas, actualmente controladas mayoritariamente por compañías transnacionales, 21 también son responsables de la disminución de la producción alimentaria.

Por último, la guerra en mayor o menor escala, provoca emigraciones forzosas con abandono de los terrenos cultivados y pérdidas de las cosechas que afectan la disponibilidad de alimentos.

\section{Accesibilidad}

La accesibilidad de los alimentos está determinada por la existencia de los mismos y las posibilidades de adquisición. La canasta básica familiar ha sufrido en los últimos años una elevación de precios en general, que afecta a los sectores de menores ingresos. Los valores del consumo promedio han sufrido una disminución en los últimos años.22 Para la mujer la inequidad de género, en materia laboral y salarial, significa una mayor dificultad para garantizar una alimentación adecuada.

Las recomendaciones diarias de aporte energético y nutrientes para la mujer establecen un promedio de $2.200 \mathrm{kcal}$, con un incremento promedio de $200 \mathrm{kcal}$ a partir del segundo trimestre de la gestación y de $500 \mathrm{kcal}$ durante la lactancia, con ingestión diaria de 46-50 gramos de proteínas que deben aumentarse a 60 en el embarazo y 65 en la lactancia, aportes ligeramente aumentados de vitaminas B, C, D y E y un considerable aumento de las necesidades de hierro, zinc y ácido fólico durante la gestación. 23

Esto sólo podría resultar paliado por una política de subsidios. En Cuba una de las formas de aliviar este problema ha sido la vinculación de las embarazadas con centros laborales que les brindan una alimentación supletoria de modo gratuito, así como el ingreso en hogares maternos y la asignación de dietas especiales tanto individualmente como a los hospitales materno-infantiles, por parte de los organismos del Estado. 24

Pese a que en la Declaración de Roma sobre Seguridad Alimentaria se establece que los alimentos no deben usarse como instrumentos de presión política o económica, Cuba ha sufrido en este aspecto los efectos del embargo norteamericano, 25 al igual que otros países como Irak.

\section{Calidad de los alimentos}

La Food and Agriculture Organization y la Organización Mundial de la Salud (OMS) han señalado la importancia de medir la calidad de las dietas en diferentes regiones para establecer las diferencias derivadas de la disponibilidad de alimentos y de los hábitos alimentarios y poder sugerir las correcciones necesarias, determinando los requerimientos básicos. La evaluación de la calidad de las proteínas por parte de los expertos se realizó en el año 1992.26

Las proteínas de la dieta constituyen un determinante ambiental para el desarrollo de los efectos del $\mathrm{CO}$ en el organismo, 27 por lo que se ha estudiado la posibilidad potencial de reducir los riesgos reproductivos que este representa utilizando suplementos proteicos en la gestación, con resultados que permiten afirmarlo. Para las mujeres del mundo subdesarrollado esto significa que el riesgo es aun mayor, ya que su alimentación es generalmente deficitaria en proteínas.

La necesidad de una agricultura sostenible y ecológicamente responsable incluye una disminución en el uso de los plaguicidas. Un estudio realizado en el quinquenio 1990-1995 en Cuba encontró niveles elevados de ditiocarbamatos en cultivos de tomate, cebolla y papa. Estas sustancias, en ensayos experimentales con ratas, han demostrado tener efectos teratogénicos. 28 La dioxina, producto contaminante que se origina en numerosos procesos industriales, ha sido otra protagonista frecuente de escándalos de repercusión mundial, al demostrarse su existencia en concentraciones elevadas en variados productos empleados en la alimentación humana. 29

Otro elemento a tener en cuenta es la venta de alimentos en las calles, por las dificultades para lograr el control de la calidad y la inocuidad de los mismos, que pueden convertirse en medios de transmisión de enfermedades. Los vendedores y los consumidores tienen perfiles socioeconómicos similares y para su selección no tienen en consideración los valores nutricionales de los productos alimenticios. 30

Un nuevo problema es la introducción de alimentos genéticamente modificados, cuyos efectos a largo plazo resultan desconocidos, y que no están sujetos a restricciones, ya que los acuerdos en la reunión de Cartagena de Indias sobre Bioseguridad fueron saboteados tanto por la Unión Europea, como por el Grupo de Miami (EEUU, Canadá, Australia, Argentina, Chile, Uruguay). 31 


\section{Cultura alimentaria}

La variable cultura parece mantener su capacidad para explicar alguno de los procesos actuales, ya que reconoce tendencias, costumbres y gustos de la población. Sin caer en el relativismo cultural, que significaría una defensa a ultranza de las tradiciones culinarias, resulta indiscutible que la población en general desconoce los principios básicos para una nutrición adecuada, que se rige más por el gusto y las posibilidades económicas que por las verdaderas necesidades de la salud. Los estilos de vida, en general, se conforman en relación con las condiciones de vida que los sustentan, pero es generalmente la mujer la elaboradora de alimentos en el hogar, por lo que su educación nutricional repercute sobre toda la familia.

Es conocido que la anemia en la mujer fértil, por ejemplo, es algo frecuente, 32 y que al principio el embarazo 33 puede resultar inquietante su magnitud por su asociación con mortalidad materna, prematuridad, bajo peso al nacer, mortalidad perinatal y disminución de la resistencia a infecciones.

Estos resultados son producto de una dieta insuficiente y desequilibrada, que puede corregirse utilizando la educación nutricional y la promoción social, analizando los hábitos alimentarios, los alimentos disponibles y su accesibilidad.

La tradicional polémica entre vegetarianos y comedores de carne ya tiene más de un siglo de existencia y la costumbre del caldo de gallina para las puérperas fueron aspectos señalados por el propio José Martí, apóstol de la independencia de Cuba, que reconocía la necesidad de la educación en materia de nutrición. 34

La utilización infundada de cualquier esquema dietético de moda por razones estéticas, siguiendo los patrones de la sociedad de consumo también constituye un peligro para la salud cuando se carece de una base racional. 35

Un problema muy particular es la necesidad de productos alimenticios que satisfagan los requerimientos de personas que sufren de trastornos metabólicos, como es el caso de los diabéticos, y de manera muy especial la gestante diabética, problema íntimamente ligado con la calidad de vida de estas pacientes, que deben asumir limitaciones para las cuales a veces no han sido preparadas y que les son impuestas sin una educación adecuada, que les permita ejercer de manera efectiva el autocuidado de la salud.

En los años 60 el Dr. Ernesto "Che" Guevara (1970: 77)36 expresó: "Muchas veces debemos cambiar todos nuestros conceptos, no solamente los conceptos generales, los conceptos sociales o filosóficos, sino también, a veces, los conceptos médicos. $Y$ veremos que no siempre las enfermedades se tratan como se trata una enfermedad en un hospital, en una gran ciudad, veremos entonces que el médico tiene que ser también agricultor, y como aprender a sembrar nuevos alimentos, y sembrar con su ejemplo, el afán de consumir nuevos alimentos".

\section{Referencias}

1. De Onis M, Blösner N, Villar J. Levels and patterns of intrauterine growth retardation in developing countries. Eur J Clin Nutr 1998; 52 Suppl: 5- 15.

2. WHO (World Health Organization). United Nations Commission on Human Rights adopts resolution and decisions on health related matters. Int Dig Health Legis 1997; 48: 429-30.

3. Amador M. Información sobre seminario Taller sobre Obesidad y Pobreza. Rev Cubana Aliment Nutr 1996; 10: 49-52.

4. Berdasco-Gómez A, Romero del Sol JM. Circunferencia del brazo como evaluadora del estado nutricional del adulto. Rev Cubana Aliment Nutr 1998; 12: 86-90.

5. He J, Klag MJ, Whelton PK, Chen JY, Qian MC, He GQ. Dietary macronutrients and blood pressure in Southwestern China. J Hipertens 1995; 13: 1267-74.

6. Obarzanek E, Velletri PA, Cutler JA. Dietary protein and blood presure. JAMA 1996; 275: 1598-1603.

7. Stamler J. Dietary salt and blood pressure: reprinted from the Third International Conference on Nutrition in Car-
dio-Cerebrovascular Diseases. Ann N Y Acad Sci 1993; 676: $122-56$.

8. Jacques PF. Effects of vitamin $\mathrm{C}$ on high-density lipoprotein cholesterol and blood pressure. J Am Coll Nutr 1992; 11: 139-44.

9. Zemel MB. Insuline resistance vs. hyperinsulinemia in hypertensión: insuline regulation of $\mathrm{Ca} 2+$ transport and $\mathrm{Ca} 2+-$ regulation of insuline sensitivity. J Nutr 1995; 125 Suppl: 1738-43.

10. Kesteloot H. Relationship between dietary cations and blood pressure. Ann Nutr Metab 1991; 35 Suppl 1: 109-18.

11. Cuba. Ministério de Salud Pública. Oficina Nacional de Estadísticas. Perfil estadístico de la mujer cubana en el umbral del siglo XXI. La Habana: Centro de Estudios Demográficos; 1999.

12. WHO (World Health Organization). Global prevalence of vitamin A deficiency. Geneva; The Organization; 1995. (WHO/NUT 95.3)

13. Rodrígez-Pita G. Acido fólico y vitamina B12 en la nutrición humana. Rev Cubana Aliment Nutr 1998; 12: 107-19. 
14. Hernández CM, Izquierdo A. Función del cinc en la recuperación inmunonutricional de lactantes malnutridos. Rev Cubana Aliment Nutr 2000; 14: 65-70.

15. Wacker J, Frühalif J, Schulz M, Chiwora FM, Volz J, Becker K. Riboflavin deficiency and preeclampsia. Obstet Gynecol 2000; 96: 38-44.

16. Wakatsuki A, Ikenoue N, Okatani Y, Shinohara K, Fukaya $\mathrm{T}$. Lipoprotein particles in preeclampsia: susceptibility to oxidative modification. Obstet Gynecol 2000; 96: 55-9.

17. FAO (Foods and Agriculture Organization), OMS (Organización Mundial de la Salud), ONU (Organización de las Naciones Unidas). Necesidades de energía y proteínas: informe de una reunión consultiva conjunta. Ginebra; The Organizations; 1985. p. 56-68. (Serie de Informes Técnicos, 724).

18. Porrata C, Hernández M, Argüelles J. Recomendaciones nutricionales y guías de alimentación para la población cubana. La Habana: Pueblo y Educación; 1996.

19. Rey-Santos O. Diez años después de Río. Rev Ci Innov Desar 2001; 6: 19-23.

20. Petinaud-Martínez J. Un archipiélago se resiste al caos. Rev Ci Innov Desar 2001; 6 : 25-9.

21. Val Giddings. A new green revolution. Rev Our Planet 1999; 10: 22-4.

22. CEPAL (Comisión Económica para América Latina). La economía cubana: reformas y desempeño en los 90 . México: Fondo de Cultura Económica; 1998.

23. Picciano MF. Embarazo y lactancia". In: Zieglery EE, Filer LJJr, editores. Conocimientos actuales sobre nutrición. 7. ed. Washington (DC): OPS; 1997. p. 410-22. (Pulicación Científica, 565).

24. Barreto J, Santana S, Martínez C, Salas AM. Grupo de apoyo nutricional hospitalario: diseño, composición y programación de actividades: guía para la práctica. Rev Cubana Aliment Nutr 2000; 14: 55-64.
25. American Association for World Health. The impact of the US embargo on health \& nutrition in Cuba. Washington (DC): The Association; 1997.

26. FAO (Foods and Agriculture Organization), OMS (Organización Mundial de la Salud). Evaluación de la calidad de las proteínas: informe de una consulta de expertos. Roma: The Organization; 1992. (Estudios FAO: alimentación y nutrición, 51).

27. Singh J. Gestational protein supplementation reduces developmental toxicity of carbon monoxide. Front Fetal Health 2001; 3: 120-2.

28. Vega LO, Arias JA, Conill T, González ML. Uso de plaguicidas en Cuba, su repercusión en el ambiente y la salud. Rev Cubana Aliment Nutr 1997; 11: 111-6.

29. Herkovits J. Efectos adversos de contaminantes orgánicos persistentes (COPs) en el período prenatal: compuestos tipo dioxinas. Arch Arg Pediatr 2000; 18: 182-7.

30. Lengomin ME, Caballero A, Monterrey P, Arcia J. Riesgos de la venta de alimentos en las calles. Rev Cubana Aliment Nutr 1997; 11: 79-83.

31. Egziabher TBG. Safety denied. Rev Our Planet 1999; 10: 22-4.

32. Gay J, Padrón M, Amador M. Prevención y control de la anemia y la deficiencia de hierro en Cuba. Rev Cubana Aliment Nutr 1995; 9: 52-61.

33. Cuba. Ministerio de Salud Pública. Informe de vigilancia nutricional materno-infantil: 1996. La Habana: El Ministerio; 1997.

34. Suardíaz ME, Espinosa E. Martí, la salud humana. Pal Nuev 2001; 93: 43-5.

35. Martin-González IC. Usos y abusos de las dietas adelgazantes. Rev Cubana Aliment Nutr 1997; 11: 123-5.

36. Guevara E. Discurso inauguración del curso de adoctrinamiento. In: Ernest "Che" Guevara: Obras, 1957-1967. La Habana: Casa de las Américas; 1970. p. 7

Recebido em 27 de novembro de 2002

Versão final reapresentada em 13 de janeiro de 2003

Aprovado em 25 de fevereiro de 2003 